\title{
Carnets
}

Revue électronique d'études françaises de l'APEF

Deuxième série - 5 | 2015

Imaginaires de guerre et autres conflits

\section{Mal fou arda Nerbona}

La violence épique : un imaginaire ambigu

\section{Mathieu Dijoux}

\section{(2) OpenEdition}

Journals

Édition électronique

URL : http://journals.openedition.org/carnets/333

DOI : $10.4000 /$ carnets.333

ISSN : 1646-7698

Éditeur

APEF

Référence électronique

Mathieu Dijoux, "Mal fou arda Nerbona », Carnets [En ligne], Deuxième série - 5 | 2015, mis en ligne le 30 novembre 2015, consulté le 19 avril 2019. URL : http://journals.openedition.org/carnets/333 ; DOI : $10.4000 /$ carnets.333

Ce document a été généré automatiquement le 19 avril 2019

\section{(c) (1) 8}

Carnets est mis à disposition selon les termes de la licence Creative Commons - Atribution - Pas d'utilisation commerciale 4.0 International. 


\title{
Mal fou arda Nerbona
}

\author{
La violence épique : un imaginaire ambigu
}

\section{Mathieu Dijoux}

Dans sa célèbre pièce 37, «Be m platz lo gais temps de pascor », Bertran de Born ne dissimule pas le plaisir que lui procure la vue des corps mutilés des guerriers morts dans la mêlée, sublime spectacle dont la jouissance surpasse, de l'aveu même du poète, le bonheur élémentaire de manger ou de boire. Quelle que soit la manière dont les commentateurs interprètent une telle célébration de la violence par le troubadour (Mancini, 1993; Gouiran, 1994), nul ne contesterait que cet enthousiasme soit étroitement solidaire de l'esthétique des chansons de geste. L'affaire semble entendue: la mort violente, dans l'épopée médiévale, est une fête dont le chevalier est le maitre de cérémonie.

2 Or, la réalité nous paraît plus complexe et nous défendons l'idée que l'imaginaire épique de la violence au Moyen Age est bien plus ambigu qu'on ne l'affirme généralement. Nous n'entendons absolument pas minimiser, par exemple, l'importance dans l'économie de la Chanson de Roland d'une « poétique du génocide joyeux » - selon l'énergique et heureuse formule de Jean-Charles Payen (1980), mais il nous paraît également important de nous montrer attentif aux dissonances présentes dans le chant.

\section{Chant et contre-chant}

3 Nous retiendrons comme point de départ de notre démonstration l'épisode de la Prise de Narbonne, tel que le rapporte la seule version vénitienne (V4), de la laisse 285 à la laisse $319^{1}$. Nous ne nous appesantirons pas outre mesure sur la modalité selon laquelle le chant intègre une matière qui lui est $a$ priori étrangère : tout porte à croire qu'il s'agisse en la circonstance d'une jonction cyclique, pour reprendre une terminologie élaborée par d'autres que nous (Heintze, 1994 et Suard, 2011). En effet, ce développement original présente à nos yeux l'intérêt majeur de constituer une véritable palinodie, manifestant au grand jour la dimension négative de la violence.

De fait, l'épisode procède à l'inversion systématique de la structure narrative de la Chanson de Roland et doit se comprendre comme un contre-chant interne. Ce travail 
d'inversion procède à un triple renversement: l'ouverture de la séquence (laisses 285-289) constitue une reprise inversée de la conclusion de la guerre en Espagne (laisse 283) et le poème semble donc suivre un cours rétrograde selon un axe de symétrie symbolisé par la laisse 284 ; le développement de la séquence (laisses 290-295), quant à lui, renverse l'ouverture du chant (laisses 14-17) ; la conclusion (laisses 296-318), enfin, forme le contrepoint du développement du poème de Roncevaux. Mais la structure narrative n'est pas la seule à être affectée, puisque l'idéologie même du chant est subvertie et que c'est en cela surtout que l'épisode mérite d'être qualifié de palinodie.

5 Respectant l'ordre de la séquence, nous considérerons en premier lieu les oppositions systématiques entre la prise de Saragosse et celle de Narbonne, en gardant à l'esprit que ces deux événements se succèdent immédiatement en V4. L'opposition est bien évidemment géographique, car la cité gouvernée par Marsile se situe au-delà de la frontière pyrénéenne alors que Narbonne se situe en-deçà de cette même frontière. Mais c'est la modalité même de la capture qui attire notre attention: Saragosse ne tombe qu'après une longue et épuisante guerre, quand Narbonne s'effondre en quelques laisses, "sença plus delae » (vers 3912), c'est-à-dire sans plus de retard que la longue prière adressée par Charles à Dieu. Ces deux déplacements demeureraient bien anecdotiques s'ils ne favorisaient une inversion ultime, en ce sens que la victoire des guerriers Francs est envisagée comme un événement malheureux. Alors que la prise de Saragosse est placée sous le signe de la foi - le narrateur rappelle au vers 3829 que « li rei cree in Deo, far vol so servisie » et la guerre s'achève par un baptême général - et d'une violence virtualisée autant que possible - la conversion fait l'économie du sang versé ${ }^{-}$la chute de Narbonne se caractérise par la frénésie meurtrière des hommes de l'empereur. De fait, bien que la cité soit prise sans coup férir grâce au miracle divin qui en a fait s'écrouler les murailles, les Francs laissent libre cours à leur brutalité sanguinaire. Le vers 3911 est particulièrement éloquent à cet égard : «Brusa les femes, les enfant ont trençé » (« Ils brûlent les femmes, ils ont mis en pièce les enfants »). La célébration de la violence purificatrice et civilisatrice, habituelle dans le chant, s'efface devant le blâme d'une action sacrilège. Le vers orphelin est à cet égard particulièrement éloquent, dans la mesure où il condamne sans appel une victoire obtenue par les champions de la sainte cause: «Mal fou arda Nerbona » («D'un mauvais feu brûla Narbonne »).

6 La violence est donc envisagée comme une souillure désormais, et la mutation est aussi radicale qu'elle est soudaine. Cette rupture se prolonge logiquement dans le deuxième mouvement, qui inverse l'ouverture du poème de Roncevaux. De fait, la chanson commence sur la compétition mettant aux prises Naimes, Roland, Olivier et Turpin : les quatre héros rivalisent d'enthousiasme pour obtenir le privilège de se rendre seul dans une ville ennemie afin de négocier la paix avec un roi perfide. Quant à elle, la séquence se poursuit par une compétition inédite dans le genre épique, car les barons de Charles rivalisent pour ne pas rester seuls dans une ville conquise et la protéger par la guerre des attaques ennemies. Après qu'un silence général a répondu à l'interrogation de Charles, désireux de savoir lequel de ses hommes désirait recevoir la ville en fief, Richard, Ogier et Hernaut, pourtant sollicités nommément par l'empereur, déclinent tour à tour une proposition que la morale héroïque eût exigé de devancer ${ }^{3}$.

7 La séquence se referme sur un ultime renversement: Hernaut propose son fils légitime pour rester seul en territoire apparemment pacifié mais en réalité hostile, tandis que Ganelon avait désigné son beau-fils pour rester seul en Espagne, terre dont la pacification n'est qu'illusoire. Si la manœuvre du traître était frauduleuse et permettait de tendre une 
embuscade à Roland, projet motivé par l'antagonisme qui oppose les deux personnages, celle de Hernaut s'explique par l'intention louable d'assurer un fief à Aymeri. Ce nonobstant, la proposition, dont le principal intéressé est ravi, suscite un antagonisme virulent entre le père et le fils. Emporté par un élan véritablement héroïque, Aymeri désire se présenter lui-même devant l'empereur et refuse obstinément que son père l'introduise en cour : le conflit dégénère au point que Hernaut affiche la ferme intention de le décapiter. La menace, fort heureusement, n'est pas mise à exécution, mais le spectre de la violence mauvaise n'en entre pas moins une nouvelle fois en scène.

L'épisode détruit donc, de manière spectaculaire, les valeurs héroïques sans lesquelles la chanson de geste perd non seulement tout sens, mais encore toute possibilité d'existence. Le génocide joyeux auquel chaque guerrier digne de ce nom rêve de contribuer devient, pendant l'espace de quelques laisses, une souillure sanglante dont les barons de Charles entendent désormais se tenir éloignés; inversement, la solidarité entre membres d'un même lignage, notion fondamentale dans l'épopée médiévale, est occultée par le motif scandaleux du parricide. La question se pose dès lors de savoir quelle portée revêt la palinodie : s'agit-il d'un simple exercice rhétorique, né de la fantaisie d'un jongleur ou d'un copiste, ou bien faut-il considérer qu'elle révèle la part d'ombre de la violence épique? Il nous semble incontestable que la prise de Narbonne permette de saisir la complexité et l'ambiguïté d'un imaginaire, trop souvent réduit à son aspect le plus évident.

\section{Le pire des Francs}

9 Si le feu mauvais de la violence brûle sous le boisseau dans la chanson de geste, les lueurs que le lecteur peut entrevoir lui permettent de saisir la nature éminemment problématique de la violence épique. La question, en effet, n'est nullement marginale ; tout au contraire, elle se situe au cœur même du genre. Dans cette perspective, la Croisade des Albigeois représente la manifestation la plus frappante d'une ambiguïté constitutive de l'épopée médiévale, dont l'auteur ou les auteurs tirent les conséquences dernières ${ }^{4}$. Pour ce qui concerne la Chanson de Roland, il est intéressant de constater que le personnage du héros éponyme incarne les contradictions de l'univers héroïque.

La chose est connue, Roland mérite le titre de meilleur des Francs en vertu de sa prouesse et son courage ${ }^{5}$. Mais ces qualités précisément, parce que les lois de l'épopée exigent que le héros les incarne à un degré excessif, sont les raisons mêmes pour lesquelles le neveu de Charles mérite aussi le titre de pire des Francs. Roland, qui fait corps, littéralement, avec son épée, est un être de pure violence, ce qui signifie nécessairement, dans l'imaginaire épique, qu'il a partie liée avec la violence impure.

11 Le sort de Durandal, selon le témoignage de V7 et C, symbolise parfaitement ce discours négatif de la chanson de geste sur la violence héroïque. Au lieu en effet de dissimuler sous lui l'arme sacrée et d'ainsi la préserver des ennemis, Roland recourt à une solution alternative :

Si a choisi un fontenel rovent,

Plein de venin et plein d'entoschement.

Deus ne fist hom des lo tens Moïsent,

S'il en bevoit, ne fust mort esrament.

Mout ert ardos et parfont et pullent.

La vint Rollant, corroços et dolent ; 
Entor lui garde, n'i choisi nulle gent.

Durendal prist par son fier hardement ;

Dedenz la jete, car la mort le sosprent ${ }^{6}$.

12 Au lieu de débattre de l'interpénétration éventuelle des traditions épique et romanesque et de convoquer le spectre de Girflet jetant Excalibur dans un lac, nous préférons nous confronter aux significations d'une telle scène. La couleur rouge du cours d'eau, son bouillonnement ainsi que la souillure qui l'emplit nous semblent renvoyer tous trois à la dimension négative de la violence. L'assurance d'une mort prochaine pour tout humain étanchant sa soif dans le "fontenel » symbolise le destin inéluctable attendant qui succombe à l'attraction de la frénésie meurtrière. Certes, le caractère inquiétant de la source et la solitude qui règne alentour offrent l'apparence d'un sanctuaire inviolable dans lequel déposer Durandal, mais ils la disqualifient pour accueillir une épée contenant, comme le rappelle le poète deux laisses plus tôt, les reliques de sainte Sophie, saint Pierre et saint Denis. Le contraste donc entre le lieu et l'objet correspond précisément aux deux faces de la violence qui, dans le poème, suscite autant de respect que d'effroi. Selon la logique qui régit en profondeur la chanson, cette source constitue une terre d'élection pour l'arme de Roland.

13 Aussi n'y a-t-il nulle surprise à constater que le neveu de Charles soit le seul personnage à être au contact d'une eau qui rebuterait tout autre que lui. Si un rapprochement devait impérativement être établi avec une autre œuvre médiévale, il y aurait quelque pertinence selon nous à considérer la Chanson de Guillaume. Au cours de son agonie, Vivien - personnage dont l'héroïsme admirable rappelle étroitement l'ardeur rolandienne - ne trouve pour se désaltérer qu'un cours d'eau fangeux, rendu impur par le sang et la cervelle des combattants morts sur le champ de bataille ${ }^{7}$. La chanson ne s'embarrasse pourtant pas de cohérence et Guillaume retrouve le corps de son neveu étendu près d'une fontaine dont les eaux limpides chantent agréablement à l'oreille (vers 1989 et 2011). Les ressources de la philologie peuvent expliquer les accidents de la transmission textuelle et permettent de discerner strates et interpolations; elles sont impuissantes à rendre compte des causes de la contradiction. Il nous semble, pour notre part, que la raison en tient à l'ambivalence constitutive du guerrier médiéval. La source impure est mentionnée dans un épisode où Vivien laisse libre cours à sa frénésie meurtrière et dévoile sa bestialité (le vers 860 précise qu'il «se redresse comme un sanglier furieux»). La description du locus amoenus, quant à elle, intervient dans une scène où Guillaume bénit son neveu; elle s'explique ainsi par la réintégration du guerrier dans la société humaine et, en l'occurrence, dans la communauté des chrétiens. Sur ce point, la rencontre entre les deux chansons de geste nous paraît concluante: Roland personnifie tout à la fois l'idéal héroïque et la souillure de la violence.

14 L'anecdote de la pomme offerte à l'empereur par son neveu conforte encore notre analyse. La critique l'a abondamment commentée, au point que le même acte a été tour à tour considéré comme une plaisanterie, un geste de séduction diabolique ou une invention du traitre jouant subtilement des peurs de l'ennemi païen, réputé connaître une prophétie légendaire turque (Jenkins, 1965 ; Brault, 1971 et 1987 ; Hunt, 1980). Aucun commentateur pourtant n'a attaché d'importance aux circonstances dans lesquelles la pomme est offerte, bien que le texte ne soit pas économe de détails révélateurs :

Er main sedeit l'emperere suz l'umbre :

Vint i ses niés, out vestüe sa brunie

E out predét dejuste Carcasonie ;

En sa main tint une vermeille pume : 
"Tenez, bel sire", dist Rollant a sun uncle,

"De trestuz reis vos present les curunes."

Li soens orgoilz le devreit ben cunfundre.

Kar chascun jur de mort sei abandunet. ${ }^{9}$

Les deux premiers vers créent en effet un contraste entre l'empereur et son neveu, en opposant l'inaction tranquille de l'un à l'ardeur guerrière de l'autre. À l'extrême limite, notre explication pourrait s'accommoder d'une lecture littérale de la chanson et admettre que le poème s'embarrasse de strictes considérations météorologiques. Il nous semble pourtant que cette retraite de Charles sous un arbre signifie autre chose que le désir, bien compréhensible, de fuir « la chaleur qui est grande en été » (comme le précise le vers 585 de V7). La fournaise espagnole n'est pas sans rapport avec le feu de la violence et de la guerre, qui brûle sempiternellement Roland, comme en témoigne le fait qu'il soit armé de sa cotte de mailles. Certes, le roi chrétien n'est pas exactement ce que l'on appelle une figure pacifique, mais l'obsession belliqueuse n'affecte, dans la chanson, que le seul Roland. V7 et C précisent d'ailleurs qu'une grande partie des Francs entourent Charlemagne, ce qui suggère la singularité de Roland.

Afin d'apprécier pleinement l'anecdote, il convient de relever que le héros vient tout juste de livrer un combat victorieux et qu'il est, par conséquent, tout empli du furor guerrier. Ceci explique sans doute qu'il puisse transgresser de manière aussi frontale l'ordre établi : bien que le guerrier dépose l'univers entier dans la main de son seigneur, son geste traduit en réalité un souverain mépris pour la fonction royale. Ivre de sa toute-puissance, le preux prétend renverser ou établir selon son bon plaisir les monarchies. Il ne se contente pas de menacer Marsile ni même les rois païens. La formule employée («trestuz reis») est particulièrement éloquente et concerne en toute rigueur la personne de Charlemagne. Bien que Roland, dans les versions de Venise-Châteauroux et de Châteauroux, exprime son intention de créer un empire universel régi par son oncle, la glose de ses propos par Ganelon met en lumière l'abolition de la fonction royale qu'implique le gab:

E tuit li roi seront desherité

E les corones lor toldra de son gré. ${ }^{10}$

\section{Heur et malheur du guerrier médiéval}

17 En ce point de notre analyse, le recours à la pensée de Georges Dumézil s'impose comme une évidence. Les deux faces, positive et négative, du héros de la chanson de geste ne sauraient en effet être justiciables de la seule interprétation psychologique. Il convient en effet de les replacer dans une perspective plus large et de les comprendre comme la manifestation d'un imaginaire de la fonction guerrière hérité de la pensée indoeuropéenne. De fait, dans l'ouvrage dont nous pastichons dans cette section le titre (1969), ce mythologue a magistralement démontré l'ambiguïté constitutive des guerriers mythiques dans l'aire indo-européenne. C'est ainsi que peut s'appréhender la complexité de la figure d'Horace : sauveur de sa cité lors du combat qu'il livre aux Curiaces, dont le sort de Rome est l'enjeu, il s'avère une menace pour son peuple lorsqu'il laisse libre cours à son furor belliqueux et qu'il tue sa sœur à l'intérieur des enceintes de la ville ${ }^{11}$. Le champion romain mérite donc la reconnaissance éternelle de son peuple et la peine de mort tout à la fois. 

antique. Son ardeur au combat en fait le défenseur privilégié de l'empire qu'il a grandement contribué à constituer, si l'on en croit le catalogue des territoires soumis par son épée. L'énumération fameuse de la laisse 172 (0) manifeste un équilibre harmonieux entre service du roi et de sa communauté et plaisir pris dans l'exercice perpétuel de la violence. L'anaphore des vers 2322, 2324, 2327 et 2331 traduit à la perfection la rencontre entre les dimensions collective et individuelle de la fonction guerrière : l'hémistiche « Jo l'en cunquis » («Par elle [Durandal] je conquis pour lui [Charlemagne]) célèbre une union rendue évidente par la juxtaposition des trois pronoms ${ }^{12}$. Roland représente une pierre angulaire de l'empire, si bien qu'il semble être le seul chevalier irremplaçable, comme l'affirme Charles au vers 840 de 0 : «Deus, se jol pert, ja n'en avrai escange!» («Dieu, si je le perds, je n'en trouverai jamais l'équivalent »). Récurrente dans l'ensemble des variantes françaises, la métaphore consistant à assimiler Roland au bras droit de Charles exprime sous la forme la plus symbolique et la plus remarquable cette harmonie entre le roi et le guerrier.

Pourtant, la tradition est témoin que le rapport entre les deux hommes est loin d'être aussi simple qu'une lecture rapide le laisserait croire. La Karlamagnus Saga propose d'ailleurs de multiples variations sur la relation fondamentalement conflictuelle qui unit l'empereur au chevalier : dans sa jeunesse encore, Roland transgresse une interdiction de son seigneur, action qui devrait lui valoir la peine de mort mais qui sera seulement sanctionnée par la punition symbolique d'avoir les ongles coupés; lors de la prise de Nobles, Roland ne respecte pas l'ordre de ne pas mettre à mort le roi Forré et Charles lui donne "un coup de son gant sur le nez au point qu'il en saigna»; dans une autre branche, Roland refuse de quitter le siège de Nobles pour affronter les Saxons et Charles l'invective puis le frappe de sorte que trois filets de sang coulent du nez du guerrier, qui se retient difficilement d'exercer une vengeance sanglante; plus tard, il compromet le succès des Francs lors d'une expédition hasardeuse; dans la huitième branche, enfin, Charles prend "conscience que Roland était cause de tout cela» [du désastre de Roncevaux $]^{13}$. Dans cette perspective, le siège de Noples semble avoir une valeur toute particulière, dans la mesure où, dans la Chanson de Roland également, il sert d'argument à Ganelon pour incriminer la frénésie meurtrière de son beau-fils qui osa contrevenir aux ordres du roi (laisses 134 de 0,182 de $\mathrm{V} 7$ et 98 de $\mathrm{P}$ ).

En somme, la scène de la pomme offerte est rien moins qu'anecdotique, puisqu'elle rappelle l'extrême fragilité du lien unissant les représentants de la première et de la deuxième fonction indo-européenne, dans l'épopée en général - que l'on songe au conflit sur lequel s'ouvre l'Iliade - et dans la chanson de geste plus précisément. Roland y apparait en effet comme une menace pour l'équilibre de l'univers civilisé, qu'il pourrait entrainer à sa suite dans le chaos de guerres sans cesse recommencées. Aussi Ganelon reformule-t-il sur un mode négatif l'éloge de Charles : si le meilleur des Francs est unique, c'est aussi parce qu'il est le pire des guerriers - nous entendons par-là le plus farouchement belliqueux - le seul qui défende le parti d'une guerre à outrance quand tous rêvent de paix. Alors que Blancandrin incrimine l'aveuglement dont les Francs font preuve pendant la guerre en Espagne, le traitre prend en effet la peine de rectifier : «Jo ne sai veirs nul hume / Ne mes Rollant $»^{14}$. L'emploi de la double négation revêt en l'occurrence une signification extrêmement forte, puisqu'elle elle souligne avec insistance la marginalité du héros. Si le travail stylistique est moins remarquable dans la variante portée par C et V7 (laisses 24), le sens demeure inchangé. Alors que Blancandrin 
incrimine les Francs en général pour leur grande fierté, qui les pousse à recourir à une violence susceptible de s'abattre sur les autres comme sur eux-mêmes, Ganelon attribue la responsabilité d'une attitude collective à son seul beau-fils : «Ce fait Rollant!» («C'est Roland qui en est cause ", vers 582 de V7).

Il nous faut insister en ce lieu sur un point essentiel : la nature problématique de la violence dans l'épopée médiévale n'est pas le produit d'une lecture cléricale ultérieure, de même que la prise en considération des propos de Ganelon ne constitue en aucun cas une trahison, fût-elle intellectuelle, comme le postule Bernard Cerquiglini (1981). Le guerrier archaïque, dès l'origine, apparaît comme une figure complexe, que la pensée chrétienne réinterprète selon les perspectives qui lui sont propres, sans créer toutefois ni effacer une ambivalence plus ancienne. Ainsi, quand Bernard de Clairvaux, jouant sur l'homonymie réputée significative entre militia et malitia, condamne sans autre forme de procès la classe guerrière dans son ensemble, Jean de Salisbury affirme que le métier des armes fut institué par Dieu et que, par conséquent, il est digne d'estime et d'approbation ${ }^{15}$. Nous retrouvons donc, dans ce débat contemporain de l'apparition des premiers manuscrits des chansons de geste, les deux faces présentées par le combattant tel que le conçoit la pensée indo-européenne. L'épopée médiévale reformule en termes chrétiens la question du statut du guerrier et de sa frénésie meurtrière, sans que son enjeu fondamental ne soit modifié.

Aussi est-il fort logique que la tradition selon laquelle Roland aurait jeté Durandal dans un cours d'eau entretienne une relation étroite avec le mythe ossète de Batradz, comme l'a démontré Joël Grisward (1969). Les données essentielles motivant la comparaison sont au nombre de quatre :

- Roland est le meilleur guerrier franc, tout comme Batradz est le plus éminent représentant de la caste guerrière des Æhsærtæggatæ.

- Roland meurt immédiatement après avoir jeté son épée dans un cours d'eau ; Batradz ne peut mourir qu'après que son épée aura été jetée dans un lac.

- Un ennemi tente en vain de s'emparer de Durandal ; les Nartes s'efforcent vainement de faire accroire à Batradz qu'ils ont jeté son épée.

- Roland jette son épée dans une eau bouillonnante et rouge ; la mer devient bouillonnante et rouge lorsque l'épée de Batradz y est réellement jetée.

Afin que l'homologie prenne son sens véritable, il convient de rappeler que le destin de Batradz semble parallèle à celui de Roland. Présenté dans certains récits comme le meilleur des Nartes, le guerrier représente en même temps une menace redoutable pour sa communauté, puisqu'il lui arrive de laisser libre cours à sa violence contre ceux qu'il protège et défend de ses armes. Le destin de son épée, comme celui de Durandal, symbolise l'ambivalence de la fonction guerrière dans l'idéologie indo-européenne, ce que permettent de comprendre les études consacrées à cette question par Georges Dumézil. Il n'est pas interdit toutefois d'interroger plus longuement l'imaginaire de la violence épique en recourant à l'anthropologie de René Girard, dont l'un des mots d'ordre aura justement été de comprendre ce qui est en jeu derrière le «nom sonore d'ambivalence " (1988: 9), si généreusement prodigué par la critique, au point de sembler le dernier mot de l'analyse. 


\section{La bête sauvage, l'autre visage du combattant épique}

Bien que la théorie mimétique ait rarement été mobilisée par les médiévistes, elle constitue à double titre une ressource précieuse pour le commentaire des chansons de geste : en effet, elle permet d'une part d'éclairer sous un jour nouveau la nature collective de la violence dans le genre et, d'autre part, de penser solidairement les questions de la violence et du double, fondamentales pour la compréhension de l'épopée médiévale ${ }^{16}$.

Le lecteur le moins attentif de la Chanson de Roland aura remarqué que le poème est saturé de représentations mimétiques, qui ont partie étroitement liée avec la violence. Tous les personnages désirent se conformer au modèle héroïque que leur propose Roland, c'est-àdire à un modèle de violence. L'illustration la plus saisissante de notre propos se rencontre sans doute dans la laisse 65 de la version de Lyon, au moment où Roland mutile le bras droit de Marsile. Chaque guerrier franc a pareillement mutilé son adversaire particulier; le coup porté par Roland a donc été répété treize mille fois et a engendré treize mille fois la même blessure. Mais le texte procède par ellipse et passe sous silence les treize mille coups; il juxtapose le coup unique et la mutilation de chaque guerrier sarrasin - procédé poétique qui permet de mettre en évidence le fonctionnement de l'imitation violente. L'adéquation est parfaite entre le geste inaugural et les répétitions simultanées qu'il entraîne, ou, pour le dire dans les termes de René Girard, « dès que les jumeaux de la violence apparaissent, ils se multiplient avec une rapidité extrême, par scissiparité, semble-t-il » $(1988: 69)$.

En l'occurrence, l'imitation violente est ouvertement envisagée sous un angle positif, car elle favorise le massacre des ennemis, exploit de nature à enthousiasmer l'auditoire. Mais elle possède souvent, dans la chanson de geste, un caractère éminemment problématique, dans la mesure où elle se manifeste sous la forme de la rivalité mimétique. Dans la Chanson des Saisnes, par exemple, la compétition pour la gloire, régime sous lequel vivent les personnages épiques, oblige Charles à traverser seul la Rune afin de rétablir son autorité malmenée par ses deux meilleurs guerriers, frustrés dans leur désir de combattre. La même compétition avait déjà conduit Bérard et Baudoin à s'affronter dans un duel qui aurait pu s'avérer fatal, bien que les deux chevaliers d'élite soient compagnons. Il n'est pas exclu que le motif du compagnonnage épique recouvre souvent une rivalité mimétique : si l'on renoue les fils épars de la tradition, du Girart de Vienne au Saragoza se devine un cycle épique fondé sur l'antagonisme virulent opposant Roland et Olivier, tous deux désireux de conquérir le titre de meilleur des Francs. La Chanson de Roland n'ignore d'ailleurs pas cette tradition, comme l'attestent les célèbres scènes du cor et le coup violent porté par Olivier à Roland. Le coup n'est certes ni volontaire ni mortel, mais il intéresse de près notre propos, en ce sens qu'il est porté par un guerrier littéralement aveuglé par sa frénésie meurtrière et son envie de vengeance ${ }^{17}$.

Or l'articulation entre violence et indifférenciation, contribution majeure de René Girard à l'histoire des idées $(1972,1982)$ permet d'appréhender avec plus de finesse l'imaginaire ambigu de l'épopée médiévale. Il importe ici de rappeler que le chemin se parcourt dans les deux sens : de vouloir imiter le modèle jusque dans son désir, le disciple devient un rival; d'accomplir des actions symétriques et de vivre toujours sur le mode de la réciprocité violente, les adversaires deviennent des jumeaux. À la croisée exacte des deux parcours surgit le monstre, hypostase du double conflictuel. 
Sidérons à la lumière de cette hypothèse le portrait des «Alemant " qui forment la quatrième échelle de Charles. Les laisses que leur consacrent $\mathrm{O}, \mathrm{V} 4$ et $\mathrm{T}(219,238$ et 171) sont purement laudatives : elles vantent des guerriers courageux, préférant la mort à la honte de la retraite. V7 et $C$ en donnent une image beaucoup plus ambiguë. Certes, leur valeur militaire continue d'être louée par Naimes :

Cil fesront bien de lor trenchant espees,

Deci as elz seront ensangletees.

Encui prenont paien tante colees,

A duel moront par cez amples valees. ${ }^{18}$

Mais, d'une part, la célébration de la bravoure importe moins que celle de la brutalité et, d'autre part, elle ne fait qu'atténuer la portée péjorative de l'introduction. Naimes commence en effet à présenter les « Alemant » à Charles comme une "gent desfaee » avant de s'étendre sur leur pilosité anormale. L'insistance du conseiller sur cette particularité physique crée une proximité étroite entre ces soldats chrétiens et les monstres sarrasins, l'assimilation des uns aux autres étant rendue possible par le statut du guerrier, problématique dans la chanson de geste.

De fait, la description des hommes de Charles ne manque pas d'éveiller dans l'esprit du lecteur l'image des berserkir: le tableau que dressent de ces guerriers mythiques les chapitres 6-7 de la Ynglingasaga pourrait parfaitement s'appliquer aux « Alemant » comme aux guerriers d'Occïant qui «n'unt soing de ëlme ne d'osberc » puisque leurs «quirs » sont durs «ensement cume fer » :

Ses hommes à lui [Odin], en revanche, allaient sans cuirasse, sauvages comme des loups ou des chiens. Ils mordaient leurs boucliers et ils étaient forts comme des ours ou des taureaux. Ils tuaient les hommes et ni le feu ni l'acier ne pouvaient rien leur faire. On appelait cela "Berserksgangr ${ }^{19}$

Le Sarrasin ne diffère pas du Franc en nature mais en degré : la relation à l'animalité n'est pas métonymique (posséder un cheval hennissant ou revêtir une peau d'ours) mais synecdochique (hennir ou avoir le cuir dur comme celui d'un ours). La constitution des jumeaux présente sur un plan anthropologique l'intérêt d'exprimer les deux visages de la violence. Il est édifiant, à cet égard, que Joseph Duggan signale au lecteur la proximité entre les «Alemant» et les berserkir par une note (p. III/502 de l'édition de référence), alors qu'il ne le fait pas pour les guerriers d'Occïant, comme si les hommes de Baligant étaient coupés de toute éternité de l'imaginaire occidental. Il est difficile, il est vrai, de ne pas succomber aux effets d'optique créés par le poème et de mettre en relation des constructions données comme antithétiques.

Dans cette perspective, la version de Paris possède le précieux avantage de proposer une synthèse des contraires. Tel est le portrait qu'elle dresse des chevaliers enrôlés dans la quatrième échelle :

Alemans orent, gens moult desmesuree ;

A vint mil furent de gent bien aesmee.

Celle compaingne fu forment aïree.

La veïssiez tante broingne saffree,

Tant bons escus, tante lance aceree

Et tant destrier a la crope triulee !

De cleres armes luist toute la contree. ${ }^{20}$

La succession des deux hémistiches finaux "gens moult desmesuree » et "de gent bien aesmee» cesse de surprendre si l'on conserve à l'esprit l'ambivalence sans cesse réaffirmée de la classe guerrière. Le glissement de l'une à l'autre épithète est fonction de 
la maîtrise ou non par les hommes d'Herman de leur fureur. " Forment aïree ", cette tribu mérite le respect, car elle est emplie de l'ardeur belliqueuse nécessaire à l'accomplissement de grands exploits, et dont l'éclat des armes est le reflet extérieur. Toutefois, et pour cette raison même, elle revêt un caractère effrayant. L'intensité de la frénésie est en soi une démesure, qui laisse présager les plus effroyables massacres. Comme l'échelle appartient aux troupes chrétiennes, la dimension positive l'emporte et le poète conclut avec emphase en chantant la beauté et la qualité des armes alémaniques. Il n'en a pas moins ouvert un gouffre dans lequel risque de disparaitre la part d'humanité des Francs. Sort le guerrier, entre la bête fauve : la capacité de métamorphose que prête aux Berserkir la tradition nordique est la traduction mythique d'un équilibre instable entre deux pôles antinomiques.

L'enthousiasme avec lequel Naimes enrôle des hommes proches de bêtes fauves dans l'armée de Charles ou, à l'inverse, les propos dépréciatifs qu'il tient sur de valeureux compagnons de bataille s'expliquent donc par l'impossibilité d'attribuer fixement une valeur positive ou négative au guerrier. La description mouvante des Alémans n'est pas sans rapport avec la complexité du personnage de Roland. Des trois épithètes qui se rapportent à cette peuplade dans $\mathrm{P}$, c'est évidemment la première qui retient notre attention. "Desmesuree " évoque inévitablement les interrogations des critiques sur l'éventuelle démesure du neveu de Charles ${ }^{21}$. Il ne saurait être question, pour nous, d'une simple coïncidence mais, au contraire, d'une convergence signifiante.

Il convient toutefois de préciser que ne figure pas dans $\mathrm{P}$ le vers fameux attesté par quatre versions : «Mielz valt mesure que ne fait estultie » (O, vers 1725 : « Mieux vaut mesure que folle témérité »). L'on pourra déplorer l'absence de l'écho interne, mais qui nierait que l'épithète "desmesuree » entre en résonance avec la tradition rolandienne? D'une part, en effet, $\mathrm{P}$ conserve l'accusation de «legerie » portée par Olivier contre Roland, et ce vers porte virtuellement en lui le premier, avec lequel il forme distique dans $\mathrm{O}, \mathrm{V} 4, \mathrm{C}$ et $\mathrm{V}^{22}$. Par-delà cette seule chanson de geste, l'utilisation de distiques similaires est fréquemment attestée dans la poésie médiévale, et il revient au même pour notre propos qu'il s'agisse là de l'influence de la Chanson de Roland ou du résultat d'un procédé mnémotechnique employé par des jongleurs ${ }^{23}$. D'autre part, et surtout, cette épithète est combinée à un second élément qui fait irrésistiblement songer à Roland. De fait, l'adjectif " aïree ", qui dans ce contexte exprime la frénésie guerrière, est un strict équivalent du " curages mult pesmes e fiers » de Roland qui anime le neveu de Charles (O, laisse 18, vers 256) et qui, à l'heure du massacre, le rapproche d'une bête fauve :

Quant voit Rollans que la bataille avra,

Tant par fu fiers que lyon resambla. ${ }^{24}$

En outre, dans V7 et $\mathrm{C}$, les deux autres versions travaillant l'ambivalence des guerriers venus «d'outre les monz», Naimes garantit la qualité de leurs coups en des termes qui, encore, évoquent Roland. La promesse d'épées ensanglantées jusqu'à la garde réactive la vantance du héros lors de la première scène du cor, répétée deux fois dans ces versions :

Anz i ferai de Durendal m'espee :

Deci q'al poing sera ensanglantee. (...)

Plus de mill colps ferrai a l'ascembler (...)

Tot en vesrez lo brant ensanglenter. ${ }^{25}$

37 La régularité des rencontres autorise à affirmer la solidarité profonde entre les gens d'Herman, la peuplade d'Occïant et le chevalier franc. Dans le cas des monstres sarrasins, la frontière entre humanité et bestialité a été abolie une fois pour toutes; pour ce qui 
concerne Roland et les "Alemant ", l'héroïsme tend à se confondre en animalité altérée de sang. Si démesure rolandienne il y a, elle réside donc dans la tentation de la métamorphose qui anime le guerrier, dans cette chevauchée héroïque et folle vers le point où la comparaison avec la bête fauve cesse d'être une figure de style et se réalise effectivement. Cette démesure n'est en rien une essence, mais constitue une tension, un élan incontrôlable et incontrôlé. Un espace est ainsi ouvert, dans lequel se déploie l'imaginaire ambigu de la violence épique. De manière rapprochée, le poète emploie le même verbe, "braire", pour traduire d'abord les cris de souffrance poussés par les chevaliers francs et sarrasins, puis exprimer les hurlements bestiaux des monstres païens (O, laisses 253 et 255) : nulle part ailleurs dans la chanson de geste ne se laisse entendre aussi distinctement le langage de la violence, tout à la fois limpide et inaudible, pathétique et inquiétant.

\section{BIBLIOGRAPHIE}

\section{Corpus primaire}

BERTRAND DE BAR-SUR-AUBE, Girard de Vienne (éd. Wolfgang van Emden, Paris : Picard, 1977).

BoDEL, Jean, La Chanson des Saisnes (éd. Anne Brasseur, Genève : Droz, 1989).

Chanson de Guillaume (éd. François Suard, Paris : Librairie générale française, 2008).

Chanson de Roland (The song of Roland: the french corpus, Joseph J. Duggan, éditeur général ; Karen Akiyama, Ian Short, Robert F. Cook et alii, Turnhout : Brepols, 2005 (trois volumes).

GUILlAUME DE TUdèLE, La Chanson de la Croisade albigeoise (éd. Eugène Martin-Chabot, Paris : Librairie générale française, 1989).

Karlamagnus Saga (La Saga de Charlemagne, éd. Daniel Lacroix, Paris, Librairie générale française, 2000).

Rolandslied (éd. Dieter Kartschoke, Stuttgart : Reclam, 1993).

Saragoza (Le Roland occitan, éd. Gérard Gouiran et Robert Lafont, Paris : Christian Bourgois, 1991).

\section{Corpus secondaire}

BOUTET, Dominique (2011). « Le sens de la mort de Roland dans la littérature des XII ${ }^{e}$ et XIII ${ }^{e}$ siècles. Chanson de Roland, Chronique de Turpin, Chronique rimée de Philippe Mousket ", in Martin Aurell et Catalina Gibbea (éds.). Chevalerie et christianisme aux XII ${ }^{e}$ et XIII ${ }^{e}$ siècles. Rennes : Presses Universitaires de Rennes.

BRAULT, Gérard (1971). « Ganelon et Roland : deux anecdotes du traittre concernant le héros », Romania, 92, 1971, pp. 392-405. 
BRAULT, Gérard (1987). «Le portrait des Sarrasins dans les chansons de geste, image projective ?", Senefiance, 21, tome 1, p. 301-311.

CERQUIGLINI, Bernard (1981). « Roland à Roncevaux, ou la trahison des clercs », Littérature, 42, pp. 40-56.

CERQUIGLINI, Bernard (1989). Éloge de la variante. Paris : Le Seuil.

CHRIST, Larry (1974). « À propos de la "démesure" de Roland : quelques propos (démesurés ?) », Olifant, 1, pp. 10-20.

DumÉZIL, Georges (1959). Les Dieux des Germains. Paris : Presses universitaires de France.

DumÉzIL, Georges (1969). Heur et malheur du guerrier : aspects mythiques de la fonction guerrière chez les Indo-européens. Paris : Presses universitaires de France.

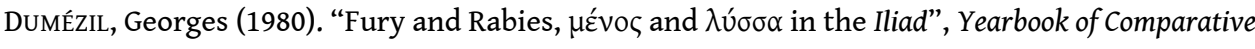
Criticism, 9, pp. 181-191.

FloRI, Jean (1984). L'essor de la chevalerie (XI-XII' siècles). Genève : Droz.

FoulET, Alfred (1958). “Is Roland guilty of desmesure?”, Romance philology, pp. 145-148.

GIRARD, René (1982). Le Bouc émissaire. Paris : Grasset.

GIRARD, René (1988). La Violence et le sacré. Paris : Hachette (Grasset, 1972 pour l'édition originale). GOUIRAN, Gérard (1994). « Bertran de Born, poète de la violence ? ", Senefiance, 34, pp. 235-251. GRISWARD, Joël (1969). « Le motif de l'épée jetée au lac : la mort d'Arthur et la mort de Batraz », Romania, 9, pp. 289-340.

HAUGEARD, Philippe (2012). « Envie, violence et sacré dans Girart de Roussillon. Lecture anthropologique et interprétation politique d'une chanson de geste ", in Hubert Heckmann et Nicolas Lenoir (éds.). Mimétisme, violence, sacré. Approche anthropologique de la littérature narrative médiévale. Orléans : Paradigme, pp. 75-96.

HeINTZE, Michael (1994). «Les techniques de la formation des cycles dans les chansons de geste », in Bart Besamusca, William P. Gerrtisen, Corry Hogettorn and Orlanda S. H. Lie (éds.). Cyclification. The development of narrative cycles in the Chansons de Geste and the Arthurians Romances. Amsterdam: Koninklijke Nederlandse Akademie van Wetenschappen Verhandelingen, pp. 21-58.

HunT, Tony (1980). “Roland's ‘Vermeille Pume”, Olifant, 7, pp. 203-211.

KAY, Sarah (1994). "The Life of the Dead Body: Death and the Sacred in the chansons de geste", Yale French Studies, 86, pp. 94-108.

MANCINI, Mario (1993). Metafora feudale: per una storia dei trovatori. Bologne : Il Mulino.

NAGY, Grégory (1994). Le Meilleur des Achéens. La fabrique du héros dans la poésie grecque archaïque. Paris : Le Seuil.

PAYEN, Jean-Charles (1980). « Une poétique du génocide joyeux : devoir de violence et plaisir de tuer dans la Chanson de Roland », Olifant, 6, pp. 226-236.

SUARD, François (2011). Guide de la chanson de geste et de sa postérité littéraire (XI $\mathrm{XV}^{e}$ ). Genève : Droz. ZumThoR, Paul (1972). Essai de poétique médiévale. Paris : Le Seuil. 


\section{NOTES}

1. Nous respectons les sigles usuellement retenus pour désigner les versions françaises de la Chanson de Roland ( $\mathrm{O}=$ Oxford ; V4 = Venise ; $\mathrm{C}=$ Châteauroux ; V7 = Venise-Châteauroux ; $\mathrm{T}=$ Cambridge ; $\mathrm{P}=$ Paris ; $\mathrm{L}=$ Lyon). Nous retenons comme édition de référence du corpus français l'édition de Duggan (2001), d'après laquelle nous citerons le texte en langue originale. Conformément aux spécificités de la poésie médiévale (Zumthor, 1972 ; Cerquiglini, 1989), nous considérerons chaque version comme aussi légitime que les autres. Sacrifiant à l'usage, nous nous réfèrerons de préférence, quand les leçons concordent, au manuscrit oxonien.

2. Les vers consacrés aux éventuels récalcitrants ne précisent pas leur sort (prison, pendaison ou bûcher), ni surtout leur nombre, dont le lecteur comprend qu'il est nécessairement infime. La violence est virtualisée, autant qu'il est possible.

3. Nous passons sous silence une seconde inversion, qui garantit toutefois que les effets de symétrie soient savamment calculés : quand, dans le chant, Ganelon est désigné paradoxalement, puisqu'il n'était nullement volontaire, le traître prend la parole de manière impromptue dans le contre-chant dans le but d'indiquer à Charles la forme idoine que devrait prendre son châtiment.

4. Nous n'entendons évidemment pas trancher ici la question de savoir si la chanson occitane fut écrite ou non à quatre mains, ni déterminer les intentions présidant à une palinodie aussi fameuse. Seul importe à notre propos le fait que le contre-chant est une potentialité de la chanson de geste.

5. La référence à l'ouvrage de Grégory Nagy (1994) consacré à l'épopée homérique n'est pas innocente, en ce sens qu'y est magistralement démontrée l'ambivalence d'Achille, meilleur et pire des Achéens tout à la fois.

6. V7, laisse 236, vers 4224-4232 : «Il a vu une source teintée de rouge, / Emplie de venin et emplie de poison. / Dieu ne fit homme depuis les temps de Moïse / Qui, s'il en eût bu, ne fût mort sur le champ. / Elle était très chaude et profonde et souillée. / Là vint Roland, irrité et souffrant ; / Il regarde autour de lui et ne voit personne. / Il saisit Durandal dans son farouche courage / Et la jette dans la source, car la mort le surprend. " Le texte de C (laisse 244, vers 4116-4124) est identique. La version de Cambridge rapporte une action similaire mais évoque simplement un « russel », une " paluz» sans autre précision (laisse 128, vers 1989 et 1991). Le terme "paluz » nous semble pourtant revêtir une valeur péjorative et devoir se traduire par «boue » plutôt que par « marais ». En ce cas, la proximité conceptuelle entre « pullent» et « paluz» confirmerait que les trois manuscrits suivent une tradition unique.

7. Vers 847-850 de l'édition établie par David Macmillan, reproduite pour partie dans Le cycle de Guillaume d'Orange (Paris, Libraire Générale Française, 1996). Les vers incriminés sont consultables aux pages 622-623.

8. Nous citons la traduction proposée par Dominique Boutet dans Le cycle de Guillaume, p. 623. La nécessité de traduire "hardi sengler " par «sanglier furieux" confirme la pertinence de notre approche fondée sur la dimension problématique de l'ardeur guerrière.

9. O, laisse 29, vers 383-390: « Hier matin, le roi siégeait à l'ombre : / Son neveu vint à lui, il avait vêtu sa brogne / Et avait fait du butin autour de Carcassonne. / En sa main il tenait une pomme vermeille : / “"Tenez, beau sire", dit Roland à son oncle, / "De tous les rois je vous présente les couronnes." / Son orgueil devrait bien le confondre, / Car chaque jour à la mort il s'abandonne. » L'anecdote est racontée en de termes proches en V4 (laisse 25), C et V7 (laisse 34). Dans le Rolantslied (vers 1839-1856), c'est Blancandrin qui rapporte la scène à laquelle il a assisté durant son ambassade.

10. V7, laisse 34, vers 594-595 : «Et tous les rois seront déshérités / Et il leur enlèvera les couronnes à son gré "». 
11. Pour les besoins de la démonstration, nous simplifions à l'extrême les données du mythe romain et ne rentrons pas dans l'analyse de la comparaison entreprise par Dumézil avec les mythes irlandais et indiens.

12. La même anaphore se retrouve en V4 (laisse 188) mais $P$ (laisse 144), T (laisse 126) et L (laisse 98) répètent toutes un hémistiche duquel Charles est exclu : «J'en ai cunquis» («Par elle, j'ai conquis »). Il est intéressant de constater que la rupture de l'équilibre passe toujours par une accentuation de la dimension individuelle, et donc problématique, de l'ardeur guerrière.

13. Nous retenons comme référence l'édition établie par Daniel Lacroix (La Saga de Charlemagne, Paris, Libraire générale française, 2000), dont nous reproduisons la traduction. Les heurts surgissent respectivement aux chapitres 39 (p.114-117) et 52 (pp.131-132) de la première branche, aux chapitres 1 (pp. 543-544) et 18 (p. 570-572) de la cinquième branche et au chapitre 39 (p. 812-814) de la huitième branche. Comme le remarque Daniel Lacroix dans l'introduction à la cinquième branche, "le véritable ennemi des Francs est en eux et Roland paraît plus que jamais mis en cause » (p. 525).

14. O, laisse 29, vers 381-382: «Je ne sache pas que cela soit vrai de nul homme / Si ce n'est de Roland ».

15. Jean Flori (1984) s'appuie sur le dialogue entre ces deux penseurs influents du XII ${ }^{\mathrm{e}}$ siècle pour démontrer combien la chevalerie forme une classe difficile à apprécier pour les hommes du Moyen Âge et, par conséquent, pour le chercheur contemporain. Dominique Boutet (2011) montre comment, à travers les siècles, la représentation littéraire du chevalier oscille entre christianisation et sécularisation.

16. Sarah Kay (1994) a insisté avec raison sur la dimension collective de la violence dans la chanson de geste, qu'elle oppose à sa dimension unilatérale dans les romans de chevalerie et les hagiographies. Philippe Haugeard a quant à lui défendu le recours à la théorie girardienne par l'importance dans les chansons de geste par « la permanence (...) d'un imaginaire de la violence collective » et par la présence remarquable du motif « à la fois anthropologique et esthétique (...) du double » (2012: 81 et 89$)$.

17. Ainsi, Olivier tranche indistinctement hommes et objets, ennemis et amis : les représailles sanglantes qu'il exerce s'accommodent de victimes indéfinies.

18. C, laisse 280, vers 5013-5016 : «Ils frapperont bien de leurs tranchantes épées, / Du haut jusqu'à la garde elles seront ensanglantées. / Ce jour les païens recevront tels coups / Qu'ils mourront de douleur dans ces larges vallées. » Les vers 5126-5129 de V7 (laisse 272) répètent de manière quasi-littérale le texte de $C$.

19. Nous citons la traduction proposée par Georges Dumézil (1959 : 43).

20. Laisse 192, vers 3443-3449 : «Ils prirent les Alamans, peuple empli de démesure ; / Il y en eut vingt mille de ce peuple honorablement respecté / Cette troupe était furieuse au plus haut degré. / Vous auriez pu voir là tant de cottes de maille d'orfroi brodées, / Tant de bons écus, tant de lances acérées, / Tant de destriers à la croupe couleur de tuile. / De leurs claires armes toute la contrée reluit.»

21. Mentionnons, outre l'article fondateur de Foulet (1958), les études de Christ (1974) et de Cerquiglini (1981).

22. Nous renvoyons respectivement aux laisses 131,142, 186 et 177 des différentes versions.

23. C'est ainsi qu'à l'entrée " estoultie ", le Dictionnaire de l'ancienne langue française de Godefroy (Paris, Libraire des Sciences, 1937-1938) comporte l'exemple très significatif des vers 4385-4386 de La Chevalerie Ogier de Danemarche : «Or me requiert par sa grant legerie, / Par sun orguel, par sa grant estoltie». L'article cite également le Roman de Brut (vers 3135-3136) et le Pèlerinage de Charlemagne (vers 628-629) où legerie est associée à folie dans la formation de distiques.

24. P, laisse 22, vers 419-420: «Quand Roland voit que la bataille aura lieu / Il devint si farouche qu'il ressembla à un lion. » Si Dumézil s'est attaché à distinguer l'aristie de la fureur des berserkir 
(1980), il n'en demeure pas moins que, dans l'Iliade déjà, la mauvaise rage représente la ligne de fuite de la fougue héroïque.

25. V7, laisse 109, vers $1850-1851$ et 110 , vers 1865 et 1868 : «Je frapperai d'abord de Durandal : / D’ici jusqu'à la poignée elle sera ensanglantée. / (...) Je frapperai plus de mille coups dans la mêlée, / (...) Vous en verrez toute la lame ensanglantée. » Voir C, laisses 118 (vers 1901-1902) et 119 (vers 1914-1920). Précisons que P comporte également cette répétition (laisses 17 et 19). La frénésie guerrière y est exprimée par une variation subtile : Roland promet la première fois que son épée sera toute ensanglantée et, la seconde fois, qu'elle sera tôt ensanglantée.

\section{RÉSUMÉS}

Fondé sur un commentaire de la prise de Narbonne telle que la rapporte la seule version vénitienne (V4) de la Chanson de Roland, le présent article entend étudier la part d'ombre de la violence que révèle la chanson de geste. Contrepoint manifeste à la célébration de la bravoure héroïque, l'épisode s'attache à condamner la froide brutalité des guerriers chrétiens et invite par là même à une réévaluation de l'imaginaire épique de la violence. De fait, dans l'ensemble des sept versions formant le corpus français de la Chanson de Roland, l'éloge des prouesses guerrières n'occulte jamais la face inquiétante de la violence. La réévaluation ici entreprise conjuguera deux approches anthropologiques qu'a priori tout oppose : les théories de Georges Dumézil et de René Girard permettent en effet d'appréhender, sous des angles dissemblables mais complémentaires, un imaginaire ambigu qui s'incarne de manière particulièrement sensible dans le personnage du héros éponyme.

By commenting the capture of Narbonne as related in the Venetian version (V4) of the Chanson de Roland only, this paper intends to study the dark side of violence as revealed by the chanson de geste. An obvious counterpoint to the celebration of heroic bravery, the episode endeavours to condemn the brutality of Christian warriors and thereby encourages the reassessment of the epic imaginary of violence. Actually, in the French corpus of the Chanson de Roland, the praise of war never conceals the disturbing face of violence. The reassessment undertaken in this paper will combine two anthropological approaches: the theories of Georges Dumézil and René Girard, although very different, enable the appraisal of an ambiguous imaginary, overtly embodied by the hero, from dissimilar but complementary angles.

\section{INDEX}

Mots-clés : chanson de geste, épopée, violence, Dumézil (Georges), Girard (René)

Keywords : chanson de geste, epic, violence, Dumézil (Georges), Girard (René)

\section{AUTEUR}

\section{MATHIEU DIJOUX}

Université Stendhal-Grenoble 3

Matthieu.Dijoux@doctorant.univ-grenoble.fr 